\title{
El conflicto y la agresión en los adolescentes
}

\author{
Magdalena Alfaro Rodríguez ${ }^{1}$
}

\section{Introducción}

En Costa Rica, se ha desatado una ola de violencia y de agresión entre los adultos, tanto en la calle como dentro de la familia. Esto, de alguna manera, ha sido asimilado o aprendido por los jóvenes y lo han trasladado a los centros de estudio.

A los(as) adolescentes, con regularidad, se les cataloga de problemáticos; sin embargo, la sociedad no ha dado la importancia requerida a esta parte de la población, y, en gran medida, ellos son el vivo reflejo de una sociedad decadente; además de que no se les ha preparado para reaccionar de manera positiva ante los conflictos, y, esto ha generado, en algunos casos, que las conductas agresivas en los(as) adolescentes en los diferentes centros educativos del país,

\footnotetext{
${ }^{1}$ Bachiller y Licenciada en Música, Magister en Educación, con mención en Docencia Universitaria.
} 
sean cada vez más comunes. Se observan situaciones diversas en las que éstos actúan con agresión o violencia. Éste es un fenómeno casi natural en su comportamiento. Para ellos(as), darse una zancadilla, usar un sobrenombre, dirigirse a otro u otra con una ofensa, es cosa de todos los días; son actitudes propias de su vida cotidiana; casi podría decirse que son actitudes que están integradas a su modus vivendi.

El ser humano puede reaccionar en forma negativa o positiva ante los conflictos; cuando éstos no son enfrentados de manera creativa, se puede caer fácilmente en situaciones de agresión o violencia.

Este trabajo contiene las definiciones que dan diferentes autores a los conceptos conflicto y agresión; asimismo, presenta algunas teorías sobre el origen de la agresión y desde estas diferentes posturas se plantea una reflexión sobre los comportamientos agresivos en los(as) adolescentes. Además del abordaje del conflicto, como coadyuvante en la construcción de relaciones más sanas en los centros educativos. Culmina con algunas notas, a manera de consideraciones finales, como elementos de reflexión sobre el tema.

\section{El conflicto}

El término conflicto tiene diferentes acepciones, las que varían de acuerdo con el contexto. Algunas de las definiciones emitidas por la Real Academia Española (2001) y que convienen a este estudio son: combate, lucha, pelea, problema, materia de discusión y, en un sentido popular, podría decirse que es desacuerdo, riña, disputa, confrontación, caos, argumento o, simplemente, pleito (Lederach, 1992). Todo ello podría utilizarse para describir diferentes situaciones de enfrentamiento, sea entre personas, grupos, instituciones o países.

El conflicto es un fenómeno social con características multidimensionales, tiene componentes tanto históricos 
como biológicos de la especie humana; está sujeto al cambio y a la transformación social y cultural de la humanidad. En la vida cotidiana, es natural e inevitable, y surge, precisamente, cuando a esos dos o más bandos en conflicto les resulta difícil ponerse de acuerdo sobre diferentes situaciones, o sobre la distribución de recursos materiales o simbólicos. Entonces, se da el conflicto, el cual es la respuesta a esas incompatibilidades percibidas.

En el contexto costarricense, la palabra conflicto tiene un significado negativo: prácticamente de algo que no tiene solución y de aquello opuesto a la armonía, a lo que debería ser, al acuerdo, a las relaciones pacíficas. Doucet (1998, p 21), dice que "la palabra conflicto, usualmente, tiene connotaciones negativas, tendemos a considerarlo como lo opuesto de cooperación, armonía, acuerdo o, incluso, paz". Sin embargo, el conflicto es una excelente oportunidad para aprender, para crecer, para ser mejores personas.

Desde el mismo momento en que una segunda persona habitó el planeta Tierra y conoció a la primera, han existido los conflictos; esto debido a la diversidad de criterios y de formas de ver y de entender el mundo. El universo entero está cargado de diferentes elementos vivos, con diferentes características y necesidades; a causa de esta diversidad de elementos es que surgen, naturalmente, las contradicciones, las cuales si fueran apreciadas de un modo natural, podrían constituirse en una forma de desarrollo para todos los seres humanos.

Existen muchas razones para que se dé un conflicto; aquí se enumeran las que los teóricos estructurales expresan y de entre ellos, especialmente, las que anota Doucet (1998), al afirmar que la organización misma de la sociedad crea las causas y las condiciones para el conflicto, y el materialismo histórico, el cual relaciona el conflicto con la organización de los medios de producción, pues esa organización 
social crea o facilita las condiciones para que se den los conflictos. Los estructuralistas no buscan explicaciones sobre la forma en que se inició el conflicto, más bien, analizan las dinámicas generales que están en juego, y analizan cómo una sociedad puede estar, más o menos, propensa a desacuerdos y hasta a brotes de violencia.

Otra causa de conflicto es la relacionada con las necesidades básicas de las personas; éstas deben ser satisfechas por todos y al no ser así, muchas veces, generan inestabilidad social, lo que puede, también, redundar en conflictos y hasta en violencia. Cuanta más necesidad haya, mayor violencia se dará. Históricamente, una de las principales causas de conflictos se encuentra en el acceso, en el deseo de control y de dominio de los territorios, así como de la posesión de los recursos naturales, materiales y económicos. Éstos son los que, generalmente, han iniciado los motivos para que se hayan dado las grandes guerras mundiales.

Aunque el conflicto es un fenómeno natural, se puede aprender a enfrentarlo, de modo que se convierta en una oportunidad para que las personas obtengan alguna enseñanza y sean mejores. En algunos momentos, estos conflictos no se resuelven totalmente, pero pueden transformarse o atenuarse. Para que esto se lleve a cabo, se necesita capacidad para negociar y para alcanzar acuerdos que tomen en cuenta los intereses y las necesidades de los implicados en el conflicto; de tal forma que no se llegue a casos extremos, en los que se presente la agresión o la violencia.

Los términos "conflicto y agresión", son las dos caras de la misma moneda. Si al conflicto no se le da un buen tratamiento, puede desembocar en un hecho violento o agresivo. El conflicto no tiene por qué convertirse en agresión; pero, en algunos casos, no se establece un diálogo entre las partes, no se expresan los sentimientos, las emociones o los pensamientos, sino que se traspasa ese delgado 
hilo que existe entre ambos. Esto sucede porque los individuos no cuentan con la capacidad (no han sido formados para ello), ni con la madurez para hacer un alto en el camino y meditar, para evitar, a toda costa, una situación de agresión o de violencia.

\section{La agresión}

Éste es un fenómeno que ha impactado a todos los seres vivos a través de la historia, y que aún sigue afectando a la humanidad. Según Hortelano (1980), el término agresión, utilizado en todas las lenguas europeo-occidentales, viene del latín aggredi (ad gradi o gradus ad, paso hacia), que significa retroceder. Y éste era el primer paso para el ataque. Agresivo significa, a su vez, el que ataca y el que camina con seguridad hacia una meta.

Desde sus inicios, la humanidad ha enfrentado situaciones de agresión y de violencia, con manifestaciones de diverso orden, tales como: agresión física, emocional, social, estructural, verbal; también de orden espiritual, mental o simbólico. Este último definido por Aguilar (2002), como la imposición de significados, valores, parámetros, conductas, etc., tomados como válidos y legítimos por el conglomerado sociocultural, que supeditan a las personas, a partir de una situación diferenciada que las discrimina, imponiendo referentes simbólico-culturales, tanto en la esfera subjetiva como en al ámbito social, que perpetúan esa condición.

Es necesario mencionar que este fenómeno puede manifestarse en cualquier nivel social; sin distinciones de color, etnia, credo, preparación o edad; por tanto, puede afectar tanto a niños como a ancianos y a profesionales como a no profesionales. La agresión ha llegado a formar parte del comportamiento humano, con lo que las personas pueden ser agredidos y/o agresores, en diferentes momentos de su vida. La agresión no es un problema exclusivo de los seres 
humanos, sino que puede darse, también, en el resto de los seres vivos, ya que, como apuntan los teóricos biológicos: es una conducta intraespecífica a favor de la conservación de la especie (Lorenz, 1976b).

Éste es un concepto polisémico y multicausal, el cual se define como una acción cuyo fin es atacar o dañar a una persona, a sí mismo o a un objeto. Este daño puede manifestarse desde la agresión depredadora, que es la provocada por la presencia de una presa natural, hasta la agresión instrumental que tiene que ver con cualquier cambio en el medio (Hortelano, 1980). En la práctica, se pueden dar diferentes tipos de agresión, entre los que se encuentran: territorial (lucha por la tierra), maternal (provocada por la proximidad de un agente amenazador de las crías), sexual (cuando existen sentimientos sádicos), entre otras. Además, es una acción potente y auto-afirmativa (Hortelano, 1980) y, en algunas ocasiones, es producida por el miedo a una persona, a un objeto o a una situación.

\section{Las teorías sobre el origen de la agresión. Algunos apor- tes en torno a los comportamientos agresivos en los ado- lescentes}

Existen varias teorías que explican el origen de la agresión, entre las que se distinguen tres grandes posturas. La primera de éstas considera que la lucha entre los seres vivos se ha dado desde siempre, dentro de ésta se encuentran diversos autores, tales como: Lorenz, Ardrey, Morris y Fromm, (Lorenz, 1976a); a éstas se les ha denominado las teorías activas, las cuales atribuyen el origen de la agresión a lo filogenético ${ }^{2}$ (Lorenz, 1976a). Éstos defienden que:

\footnotetext{
${ }^{2}$ La filogenia (del griego: phylon = tribu, raza y genetikos = relativo al nacimiento, de génesis = nacimiento) es la historia evolutiva de los taxones, es decir, los grupos de la clasificación de los seres vivos. El análisis filogenético es el estudio de las relaciones evolutivas entre las distintas especies y de su reconstrucción, representada en forma de árbol filogenético.
} 
La agresión está determinada por la herencia, como instinto transmitido por los ancestros y la explican como pulsión, entendiendo ésta como impulso o instinto de todo ser vivo que busca una forma de autoprotección y/o de protección a la especie.

Cada animal en su especie, se las ha ingeniado para defender su espacio, su alimento, su territorialidad y, hasta, su pareja. Se produce, entonces, una serie de situaciones conflictivas, o agresivas que pueden manifestarse desde un roce hasta una lucha sangrienta entre dos o más seres vivos y/o entre dos o más naciones, como es el caso de las guerras, en las que las personas se exterminan unas a otras, sin piedad.

Fromm (1974) considera la agresión como un fenómeno, biológicamente, adaptativo y al servicio de la vida, como un tipo de agresión a la que el ser vivo está filogenéticamente programado, y como un fenómeno existente, tanto en los animales como en los seres humanos. Con base en esto, se tendrían que realizar los estudios correspondientes para poder afirmar que la agresión en los adolescentes obedece a razones de origen filogenético.

La segunda postura sostiene que los comportamientos agresivos son generados tanto por aspectos genéticos como ambientales. Esta posición parte de que el ser humano es complejo, es un ser integral y, por tanto, su comportamiento es la mezcla de muchos factores, tanto endógenos como exógenos. El ser humano es producto de elementos tanto genéticos como ambientales, los que interactúan en forma dinámica (Hortelano, 1980). Esta postura, tampoco podría afirmarse en el tema abordado, ya que se necesitaría realizar una serie de investigaciones, en las que se relacionen dichos factores, para poder afirmar que los comportamientos agresivos en los adolescentes están asociados a estas dos variables. 
La tercera postura sostiene que los comportamientos agresivos se aprenden y son producto del ambiente; posición que para efectos de este análisis es considerada la más pertinente, ya que los comportamientos humanos son aprehendidos y construidos socialmente, y los(as) adolescentes reproducen lo que viven en el marco de las relaciones sociales. Dentro de ésta se encuentran: Engels, Reynols, Asrin, Hutchinson y Skiner. Al respecto, Bandura (1980) dice que:

Las personas no nacen con repertorios prefabricados de conducta agresiva; deben aprenderlos de una u otra manera. Algunas formas elementales de agresión pueden perfeccionarse con un mínimo de enseñanza, pero las actividades de índole más agresiva -sea entablar un duelo con navajas, liarse a golpes con un enemigo, combatir como soldado o aplicar el ridículo como venganza- exigen el dominio de destrezas difíciles que, a su vez requieren de extenso aprendizaje. Las personas pueden adquirir estilos agresivos de conducta, ya sea por observación de modelos agresivos o por la experiencia directa del combate $(310$ y 312$)$.

Berger y Lukmann (1984), anotan que el orden social es una creación humana, es un proceso de origen humano, que se exterioriza e institucionaliza. Consideran que los hombres producen un ambiente social y que el organismo humano carece de medios, en el nivel biológico, para lograr la estabilidad en su comportamiento; por lo que el ambiente en que se desarrolle la persona es determinante en su conducta. Bandura (1980, p. 18), anota que "la violencia, en el ser humano, no es un fenómeno individual, sino un fenómeno social, y que tal violencia arranca, originalmente, del sistema y no del individuo". 
El mundo está cargado de agresión y de violencia, de relaciones interpersonales y familiares disfuncionales. La influencia de los multimedia satura a los jóvenes de películas, de dibujos animados, de novelas y de música cargados de violencia. Problemas en todos los niveles (político, económico, moral y espiritual), en el ámbito social, se constituyen en motivos suficientes para que ese orden social se vea alterado y, por tanto, broten situaciones de agresión o de violencia. Los adolescentes no están al margen de esto; ellos son vulnerables, ya que están en proceso de conformación de su personalidad; proceso en el que también definen los roles que en el ámbito social deberán desempeñar.

En la actualidad, los hechos agresivos y violentos son cada vez más comunes en las diferentes instituciones educativas de nuestro país. Algunos(as) adolescentes han tomado la justicia en sus manos; se dan de golpes con otro u otra, sin ningún miramiento, se han atacado, y, en algunos casos se han provocado lesiones graves; también han maltratado y herido a profesores, y hasta han cobrado la vida de otros seres humanos. Aunque exista un reglamento de disciplina en el Ministerio de Educación Pública en Costa Rica, éste no impide que se dé este tipo de situaciones. Esta problemática va más allá de los reglamentos o de las medidas policíacas de revisión de bultos, para decomisar todo tipo de armas; sobre todo, cuchillos, cuchillas y cutters, promovidas por el Ministerio de Educación Pública, según disposición del 25 de enero de 2005. Los alumnos dicen que "la violencia se ha convertido en una forma habitual dentro del colegio; se sienten inseguros y, además, poco protegidos por los profesores" (Varela, 2006).

Algunos ejemplos de esto los tenemos en algunas instituciones de educación secundaria: la detonación de cuatro bombas caseras en el Colegio de Rincón Grande de Pavas, hecho ocurrido el 4 de abril de 2006. Anteriormente, 
dos alumnas habían resultado heridas de bala el 16 de febrero de 2004, al dispararse un arma que portaba un compañero, entre otros actos violentos. Se dice (algunos creen que se exagera) que son, aproximadamente, doscientas instituciones las que están en alto riesgo (Ventaja, 15 de mayo, 2006). El mayor problema es que, en el interior de los centros educativos, no se cuenta con suficientes criterios formativos e informativos, acerca de cómo enfrentar los conflictos en el aula y, así, evitar o minimizar estos hechos violentos.

El individuo objetiviza la realidad por medio del lenguaje (Berger y Lukmann 1984); entonces, se puede decir que el ser humano aprehende esa realidad en todo lo que el lenguaje le proporciona. Aquí no sólo se habla de lenguaje verbal, sino también del visual, del auditivo y del gestual, los cuales indican al individuo cuál ha de ser su comportamiento, sobre todo, dentro de la familia. Algunas veces, se repite el patrón aprendido, pues todo lo que está alrededor del ser humano va a tener una gran incidencia en el comportamiento de las personas. Así pues, se aprende la agresión mediante lo que se vive dentro de las familias, lo que se ve en la televisión y en la Internet, así como en otras actividades, las que, poco a poco, se han institucionalizado y se reflejan en las diferentes formas de resolver los conflictos, tanto individuales como sociales.

Por otro lado, también existen actitudes, que a simple vista no parecieran agresivas, sin embargo, son nocivas en la construcción de la personalidad del adolescente, a la vez que fungen como aguafuertes de la ola de violencia que se vive en nuestra sociedad. Dentro de éstas se encuentran todas aquellas que en la vida cotidiana se traducen, a menudo, en palabras obscenas, saludos con un golpe, celebraciones de cumpleaños con una guerra de huevos. Ellos y ellas ven como algo natural que, constantemente, se dirijan a 
los demás con expresiones insultantes o vulgares. Asimismo, estas actitudes ofensivas se manifiestan cuando rayan las paredes, sobre todo, las de los servicios sanitarios. En ellas, se expone toda clase de insultos, y hasta se crea una especie de correo, en el que se maltratan y se dicen cómo van a castigarse. Se dan rencillas; algunos se dejan de hablar, otros se ofenden en cualquier lugar (incluso en el aula) y delante de quien sea, se hacen zancadillas, se dan de puntapiés, se amenazan, se roban, entre muchos otros actos.

La agresión es una construcción social, aprendida y sustentada, tanto en los comportamientos que a escala social se llegan a institucionalizar (adquiridos de generación en generación), como a través de los medios de comunicación. Éstos presentan diversos programas disfrazados de diversión, pero que promueven, entre otras cosas, la de-sensibilización ante la violencia. Se empieza a ver como algo normal y, así, se interioriza en la mente (subconsciente) de los jóvenes (Levine, 1997).

Algunas veces, para lograr lo que se quiere o se necesita, se podrían adoptar actitudes que van en perjuicio de los demás. Lorenz (1976b) apunta que en la organización social es necesario el principio del orden, una vida organizada en categorías, en relación con los animales superiores, en la que cada uno se preocupa siempre por mejorar su posición dentro de esta jerarquía, con lo que se crea una situación de tensión entre los individuos. Así, algunos podrían usar a una persona para subir de estatus, sin tomar en cuenta que con esto, tal vez, puede dañar a la otra persona. En el caso de los(as) adolescentes, en ocasiones, los más grandes del colegio se aprovechan de los más pequeños: les quitan la merienda, los mandan a comprar algo a la soda o los obligan a hacer alguna otra cosa, que los aminora o los maltrata.

La agresión surge en forma espontánea, por ejemplo, cuando se invade el espacio ajeno, o se dice a otro u otra 
algo que molesta, $y$, sin darse cuenta, se reacciona para el ataque. La ofensa surge no sólo por causa de situaciones externas, sino que, también, es provocada por situaciones que acontecen dentro del ser humano y no están resueltas.

En el orden social, el ser humano desea y espera tener calidad de vida, estatus y espacio no sólo físico, sino también emocional, para sentirse a gusto; sin embargo, muchas veces, estas expectativas no se cumplen por muy diversos motivos. Esto le trae, al individuo, incomodidad y podría provocar, de alguna manera, actitudes agresivas o violentas. En la adolescencia, las necesidades son apremiantes; tales como: la necesidad de aceptación, de amor, y de verse y sentirse bien. La preocupación por su autoimagen corporal, por la autoestima, por el autoconcepto, por la formación de su identidad. Además, los adolescentes, actualmente, enfrentan muchas situaciones que provocan conductas agresivas, tales como el ultraje que viven en sus hogares, la soledad, la falta de amor y el abandono por parte de sus padres y madres, el incursionar en la ingesta de diferentes tipos de drogas, y en algunos casos también a problemas de depresión, entre otras.

Otros elementos detonantes de violencia, anotados por el programa "Valor joven", son la falta de identidad, la dualidad en el discurso de los adultos, la expulsión social y la ausencia de sentido de pertenencia que los jóvenes enfrentan en la sociedad actual (citados por Munduarte; Chinchilla; Pérez y Méndez, 16 de mayo, 2006). La presión social y cultural es muy fuerte, ya que, por un lado, les asigna estereotipos y les pide estándares y niveles de comportamiento, para los que, muchas veces, no están preparados. Por su parte, la UNICEF (citado por Munduarte et al.), dice que otras causas, son la exclusión, la pobreza, la subordinación, la falta de oportunidades, la dependencia, los conflictos intergeneracionales, la inequidad, la explotación, las 
sensaciones de inseguridad. Otras causas expuestas por el PANI, y consideradas como motivo de violencia manifestadas por los adolescentes, son el enojo, los modelajes agresivos, la contrastación de la fantasía con la realidad, los comportamientos de masas (Munduarte et al.).

\section{El abordaje del conflicto}

El abordaje del conflicto no ha sido un tema de gran relevancia en el currículo escolar, por tanto, los(as) adolescentes no saben cómo enfrentarlo, no lo entienden, no están en capacidad de abordarlo en forma creativa; además, culturalmente, se les ha enseñado a ver el conflicto como algo negativo, como algo que se debe evitar a toda costa. A menudo, los conflictos o desacuerdos que enfrentan, diariamente, los(as) adolescentes, se convierten en escenas agresivas o violentas por un mal manejo de éstos. No se han establecido puentes para que los resuelvan o enfrenten de una manera positiva; lo que evitaría, así, el llegar a situaciones extremas. Los psicólogos de la Universidad Católica, quienes han realizado una investigación sobre la violencia en centros de educación secundaria, opinan que uno de los detonantes de esta ola de violencia es la forma en que se canaliza y se maneja ésta dentro de las aulas, ellos creen que se debería ir más allá de estrategias represivas (Varela, 2006).

El punto importante aquí es cómo se debe enfrentar el conflicto y no que se quiera obviar o evitar, lo cual es una idea equivocada en algunos, que creen que para que haya relaciones armoniosas no deben existir los conflictos; consideran que ni siquiera debería mencionarse esta palabra, y se olvidan que son parte de la vida y que es inevitable que ocurran. El conflicto, visto de esta forma, permite, entre otras cosas, tolerar el pensamiento divergente y respetar a 
los demás, elementos fundamentales de toda relación interpersonal sana. Así, se aprende a ceder, en algunos momentos, a entender otros puntos de vista, a compartir, a tolerar; en fin, a ver el conflicto como a un aliado y no como a un enemigo; a verlo de forma constructiva y creativa, para que, en la medida de lo posible, se pueda resolver $o$, por lo menos, se transforme.

"No podemos, simplemente, enseñar a los jóvenes que la violencia no es la manera de resolver los problemas, si continuamos recurriendo a ella para obtener nuestros objetivos y, a menudo, controlarlos a ellos" (Bandura, 1980, p. 309). Es de vital importancia, que en el ámbito educativo, los docentes traten estos temas, con un criterio que vaya más allá de definiciones teóricas. Se deben promover espacios para llevar a la práctica las resoluciones o transformaciones del conflicto, mediante la utilización de estrategias acordes con las características de los adolescentes, para, así, cooperar con la construcción de una sociedad que pueda mostrar relaciones más sanas entre sus habitantes.

Debe haber más interés, en los(as) docentes por el desarrollo moral, personal e integral de los jóvenes, es decir, por ayudarlos a ser mejores, y no sólo por llenarlos de contenidos, sobre todo, en el marco de una sociedad consumista, individualista y globalizada, como es ésta. Los valores que se deben trabajar con los jóvenes y sobre los que se debe hacer énfasis son aquellos que les ayuden a enfrentar los conflictos en forma creativa; entre los que se podrían trabajar están: el respeto, la solidaridad, el amor, la diversidad y la paz, entre otros. Es necesario analizar las formas de reaccionar ante las diferentes situaciones de la vida cotidiana, pues los jóvenes no saben cómo responder de forma positiva ante la diversidad de opiniones y de formas de entender la vida; y provocan roces, problemas o conflictos, los que coexisten en las relaciones interpersonales cotidianas. 
Es importante que se ofrezcan talleres y charlas sobre los procesos de negociación y de conciliación, como elementos fundamentales para enfrentar los conflictos en forma constructiva. Respecto a esto, Doucet (1998) anota que "un proceso de negociación puede incluir algunos de los siguientes elementos:

- Comprensión de las causas del conflicto

- Sentido de posesión del proceso de paz

- Identificación de todas las personas y grupos involucrados

- Identificación de los facilitadotes

- Fijación de un cronograma realista

- Mantenimiento del esfuerzo

- Evaluación de los aciertos y las fallas

- Grupos estratégicos" (p. 48).

En relación con los procesos de conciliación, éstos se dan cuando existe la intervención de un tercero en el proceso de análisis y transformación del conflicto. Las acciones deben ser muy claras, tales como: identificar los aspectos principales, minimizar la tensión y estimular a las partes para la interacción directa, a manera de una negociación para solución o, en su defecto, la transformación de las diferencias (Doucet, 1998).

La resolución de conflictos, como opción para evitar la agresión en los adolescentes, está enmarcada dentro de la Educación para la Paz, en la que, según Doucet (1998, p. 59): "(...) los encargados de la intervención no son expertos en el tema, sino que se involucran en un proceso mediante el cual se descubre la utilidad de la experiencia colectiva disponible en una cultura determinada. Esto constituye una gran ayuda para las partes en conflicto; a los participantes los involucra en el diseño de un proceso que 
transforme su propio conflicto de una experiencia violenta en algo más constructivo".

Es de vital importancia hacer conciencia en todas aquellas personas que se dedican a la educación para que, en el desarrollo de su trabajo, hagan una lectura de la realidad social, de los factores de riesgo y de las posibles causas de violencia en las aulas, y hacer un frente común con los padres y las madres de familia, así como con la comunidad, tanto local como nacional, para contrarrestar esa ola de violencia que afecta a todas las personas, en todos los niveles y en todas las regiones. Ya es hora de que los educadores y las educadoras dejen de conformarse con abarcar los contenidos, para cumplir con un programa. Que dejen de interesarse sólo por lo cognitivo, y dejen de poner la excusa de que: "No hay tiempo". En este sentido, anota Varela (citado por Méndez, en Munduarte et al., 16 de mayo, 2006) que "no podemos seguir insistiendo en que sólo la familia y los medios de comunicación son responsables, y que la escuela no puede hacer nada. Todos tenemos que asumir un papel activo contra la violencia".

Es necesario formar a los(as) adolescentes en valores tan importantes como la solidaridad, el amor, la justicia y la paz. Aunque los valores están contemplados dentro de los temas transversales, en los programas del Ministerio de Educación Pública, no es suficiente, pues en muchas ocasiones se quedan en el papel. Se requiere de cambios profundos en la conceptualización y en la operacionalización de esos temas.

El papel de los educadores es fundamental en la construcción de una sociedad que pueda reflejar las manifestaciones de la justicia y la paz en todos los ámbitos; con esto ayudarían a promover el desarrollo moral de los jóvenes. Al respecto, Pío XII (citado por Hortelano, 1979, p. 11), en su mensaje del 23 de mayo de 1952, dice que: "Los niños y los adolescentes tienen derecho a que se les estimule a apreciar, 
con recta conciencia, los valores morales y a prestarles su adhesión personal y, también, que se les estimule a conocer y a amar a Dios. Ruega, pues, encarecidamente, a todos los que gobiernan los pueblos o están al frente de la educación, que procuren que nunca sea privada la juventud de este sagrado derecho".

Es necesario que en todas las disciplinas que se imparten en las instituciones educativas de secundaria, se promuevan espacios de reflexión y de análisis sobre las problemáticas de la adolescencia, escuchar a los(as) jóvenes, entender cuáles son sus inquietudes, sus sueños y sus frustraciones, que puedan decir lo que les molesta, aunque a veces resulte doloroso o preocupante; de modo que, en el momento en que se presente algún tipo de conflicto, los(as) educadores(as) puedan fungir como mediadores, y, así, tratar de evitar situaciones agresivas o violentas. Posiciones como éstas son las que han estado ausentes en el abordaje de los conflictos que en los últimos años se han dado en las diferentes instituciones educativas del país, tales como el Liceo Mauro Fernández, de Curridabat, Liceo del Sur, de Moravia, Liceo de Costa Rica y, recientemente, en el de Pavas, entre otros, pues, "los educadores han optado por no mediar entre los alumnos cuando discuten de manera violenta, por miedo a las represalias o porque es mejor no meterse. Nadie adopta un papel responsable y activo para frenar la violencia" (Varela, 2006, p. 18).

Es urgente que el Ministerio de Educación Pública promueva capacitaciones en materia de: transformación de conflictos, educación para la paz y la no violencia, y se incluyan, además, algunos temas de los tratados por Lederach y Chupp (1997), tales como: la violencia y no-violencia activa, concepto y análisis del conflicto, autoestima y estilos personales, la comunicación asertiva, entre otros. Estas guías y estrategias pueden ser de gran utilidad en el abordaje 
del conflicto. Los educadores y las educadoras se convertirían, así, en verdaderos actores sociales, capaces de transformar la realidad que les ha tocado vivir.

Algunos otros planteamientos, como forma constructiva para enfrentar los conflictos, son los que se proponen en "Valor Joven" (Munduarte et al., 16 de mayo, 2006), con la realización de proyectos de expresión juvenil, mediante las artes, de la literatura u otro tipo de expresión. También deben fortalecerse y dar a conocer los programas que trabajan por la juventud, como por ejemplo la Red Nacional de jóvenes para la prevención de la violencia, promovido por la Dirección Nacional de Prevención de la Violencia y el Delito (DINAPREVI), del Ministerio de Justicia y Gracia de Costa Rica. Este programa tiene entre sus objetivos: "Fomentar la existencia de un espacio de reflexión, formación y participación continuas para los y las integrantes de la Red, y promover la formulación y la ejecución de proyectos preventivos, sobre diferentes manifestaciones de violencia, al interior de los centros educativos" (Munduarte et al.).

\section{Consideraciones finales}

En una sociedad globalizada, en la que lo que predomina es el materialismo y el individualismo, se hace necesario cambiar el rumbo de los objetivos fundamentales en materia educativa. La educación se debe centrar en el desarrollo humano, desde cada uno y cada una, y orientar todos los esfuerzos para formar una persona creativa y sensible, que pueda hacerle frente a todos los desafíos que se tienen al convivir en una sociedad con problemas, no sólo en los niveles económico, espiritual y ecológico, sino también en sus valores Es necesario que la educación se oriente a la formación de personas capaces de conmoverse ante el dolor 
humano, de solidarizarse con su vecino y de trabajar, en forma cooperativa, por el bien común y el de la naturaleza.

Dentro de todo este contexto tan complejo como el que se vive a inicios del siglo XXI, resulta fundamental seguir creyendo en el ser humano, y seguir anhelando individuos más justos, más equitativos y que construyan una sociedad que disfrute de la paz; entendiendo ésta no como la ausencia de guerra, sino como el bienestar de todos y de todas, en sus diversas manifestaciones y en los campos económico, social, político, emocional y espiritual.

Es urgente que el Ministerio de Educación Pública tome en cuenta el eje transversal de la educación para la paz, no como un discurso más, sino como una forma de vida, como parte del quehacer de las instituciones educativas; que se considere como un elemento fundament $s^{\prime}$ para poder cambiar la situación de violencia que se está dando y no llegar a la destrucción del hombre por el hombre, como una forma de satisfacer a la fuerza, las necesidades básicas de algunos que no quieren esforzarse. Debe darse prioridad al desarrollo de los valores en los adolescentes, para que sean capaces de relacionarse en forma sana, de decirse las cosas que les molesta sin herirse, de escuchar lo que los otros quieren decirles, de respetar las pertenencias ajenas, de perdonar y de aprender a retribuir, cuando sea necesario. En fin, que sea una educación que se oriente al desarrollo de las inteligencias interpersonal (Gardner, 1995), emocional (Goleman, 2003) y espiritual (Zohar y Marshall, 2001).

También se debe analizar las reacciones ante el conflicto, y cuáles podrían ser los mejores caminos para solucionarlo y tener una convivencia pacífica. De esta forma, se estaría ayudando con la construcción de una sociedad más justa, en la que no es que no vayan a existir conflictos, pero sí en la que sus habitantes puedan tener una mejor actitud ante las situaciones conflictivas, de modo que éstas 
puedan transformarse y que no se conviertan en hechos violentos.

Costa Rica, a pesar de que no tiene ejército, está cargada de violencia. Los actos delictivos que se han dado en los últimos tiempos dan mucho que pensar, este país ya no es la 'Suiza centroamericana', a la que muchos se referían; idea que aún se promueve en el extranjero. La responsabilidad de volver a una cultura de paz es de todos sus habitantes, especialmente de los educadores, quienes, día a día, pueden poner un granito de arena en la construcción de una sociedad que sea capaz de resolver los conflictos con diálogo, comprensión y consenso; y en la que los procesos de mediación y de conciliación se conviertan en una de las principales formas de acceder a los conflictos, como parte de las principales características de nuestra idiosincracia, y no como un discurso más. Que los(as) docentes no dejen de cumplir con la responsabilidad que como educadores les corresponde. Desde las altas esferas, en conjunto con todos los miembros de la sociedad, se debe trabajar por una sociedad más equitativa y justa, en la que los niveles de agresión y de violencia sean minimizados, ya que este fenómeno está ocasionando grandes estragos en la población.

Para que un grupo social mantenga la paz se requiere de la confluencia de varios factores, entre los más importantes están: la justicia, la equidad y que las necesidades básicas de todos sus integrantes sean suplidas. Además, es fundamental que el gobierno central, por medio de los organismos estatales vele por mantener el orden social. Dentro del contexto globalizado, algunos valores, tales como la solidaridad y el humanismo, se han visto menoscabados; por lo que, debe procurarse la reconstrucción de una sociedad que refleje la paz, en su sentido más amplio, y que repudie, de forma enfática, los hechos violentos y agresivos en todos sus niveles y manifestaciones. 


\section{Referencias}

Aguilar, V. (2002). La violencia simbólica sobre el género femenino en el proceso de enseñanza-aprendizaje [sic] del Derecho Penal. Tesis sometida a la consideración de la comisión de Programa de Posgrado en Estudios de la mujer para optar al grado de Magíster Scientice. Ciudad Universitaria Rodrigo Facio, San José, Costa Rica.

Bandura, A. (1980). Modificación de la conducta. México: Editorial Trillas.

Berger, P. y Luckmann, T. (1984). La construcción social de la realidad. Buenos Aires: Editorial Amorrortu.

Doucet, 1. (Ed.) (1998). Buscando la paz del mundo. Manual de recursos para la transformación del conflicto. Bogotá: Editorial Semilla.

Fromm, E. (1974). La anatomía de la destructividad humana. México: Siglo XXI.

Gardner, H. (1995). Inteligencias múltiples. Barcelona: Ediciones Paidós.

Goleman, D. (2003). La inteligencia emocional. (2ª Reimpres.).

México: Editorial Punto de lectura.

Hortelano, A. (1979). Introducción a la teología moral. La conciencia moral. Tomo I Problemas actuales de moral. Salamanca: Ediciones Sígueme.

Hortelano, A. (1980). La violencia el amor y la sexualidad. Tomo II. Problemas actuales de moral. Salamanca: Ediciones Sígueme.

Lederach, J. y Chupp, M. (1997). Conflicto y violencia. Busquemos alternativas creativas. Santa Fe de Bogotá: Editorial Semilla.

Lederach, J. (1992). Enredos, pleitos y problemas. (2ª impresión). Guatemala: Editorial Semilla.

Levine, M. (1997). La violencia en los medios de comunicación. Cómo afecta al desarrollo de los niños y adolescentes. Bogotá: Editorial Norma.

Lorenz, K. (1976a). Biología del comportamiento: raíces instintivas de la agresión. México: Editorial Siglo XXI.

Lorenz, K. (1976b). Sobre la agresión: el pretendido mal. México: Editorial Siglo XXI.

Munduarte, C.; Chinchilla, L.; Pérez M., y Méndez, J. (2006, mayo, 16). Violencia de adolescentes y jóvenes en centros de educación secundaria. Mesa redonda, Universidad de Costa Rica. 
Real Academia Española. (2001). Diccionario de la lengua española. Madrid: Editorial Espasa Calpe.

Varela, I. (2006, abril, 2). Por aulas seguras. La Nación. Suplemento Proa, p.18.

Ventaja, C. (2006, mayo, 15). Colegios incumplen orden de revisión diaria de bultos. La Nación. p. 4A.

Zohar, D. y Marshall, I. (2001). Inteligencia espiritual. Barcelona: Plaza \& Janés editores. 